\title{
Quantitative magnetic force microscopy analysis of the magnetization process in nanowire arrays
}

\author{
A. Asenjo, ${ }^{a)}$ M. Jaafar, D. Navas, and M. Vázquez \\ Instituto de Ciencia de Materiales-CSIC, Campus de Cantoblanco, 28049 Madrid, Spain
}

(Received 19 December 2005; accepted 6 June 2006; published online 27 July 2006)

\begin{abstract}
Magnetic force microscopy (MFM) imaging is a useful technique to locally study the magnetic state of nanostructures. In this paper, we have used the MFM to characterize an ordered array of $\mathrm{Ni}$ nanowires embedded in porous membrane. Due to the large aspect ratio of the wires $(30 \mathrm{~nm}$ diameter and $1000 \mathrm{~nm}$ length) they present an axial easy axis. Considering the nanowires as nearly single-domain structures and calculating the amount of wires pointing to each direction, we can obtain the average magnetization. An alternative method to analyze the MFM data is here introduced considering the distribution functions of magnetic contrast. By using this method, the magnetization process of the nanowire array is studied and the results are compared with major and minor hysteresis loops measured by superconducting quantum interference device magnetometer.

(C) 2006 American Institute of Physics. [DOI: 10.1063/1.2221519]
\end{abstract}

\section{INTRODUCTION}

Magnetic force microscopy imaging ${ }^{1,2}$ reveals as a useful tool to analyze the local magnetic behavior of the materials. ${ }^{3}$ In some cases, the magnetic force microscopy (MFM) images complete the magnetic characterization performed by standard macroscopic methods such as superconducting quantum interference device (SQUID) or vibrating sample magnetometer (VSM). In the case of nanometric devices, only few techniques can supply information about the behavior of individual entity and among them, MFM presents the highest resolution and provides simultaneously information about the topography and the magnetization process. $^{4}$

Along the last decade, the controlled fabrication and characterization of small entities have attracted the scientific community. In particular, much attention is paid to the study of magnetic nanowires since they present phenomena related to nanoscale and they have potential applications in nanotechnology. Anodization processes to achieve self-ordered nanopores in membranes have been proven to be a direct, simple, nonexpensive technique to fabricate templates for highly ordered densely packed arrays of magnetic nanowires. ${ }^{5,6}$ Such arrays of nanowires are considered excellent candidates for functionalization of membranes in a number of sensing applications. ${ }^{7-9}$

The magnetic behavior of these arrays of nanowires has been extensively studied by SQUID or VSM magnetometries where information of an array as a whole is obtained. Some aspects are nevertheless not yet fully determined as the magnetization reversal mechanism of nanowires and even their domain structure both depend on geometric dimensions. ${ }^{10-16}$ Notice that the diameter of these nanostructures $D$ is comparable or smaller than the domain wall width $\lambda_{w}$ smaller than $30 \mathrm{~nm}$ for Ni wires. ${ }^{17}$ Hence, the aspect ratio $R=L / D(L$ is the length of the wires), the material of the wires, the order-

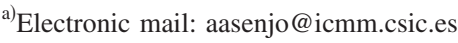

ing degree, and the lattice parameter of their hexagonal arrangement determine whether the nanowires are single or multidomains, the importance of the interaction between nanowires, and the reversal magnetization mode (curling, coherent, or nucleation).

The MFM images have been obtained by imaging the surface of the membrane where the magnetic nanowires are perpendicularly embedded. It is to be emphasized that MFM imaging informs us of the magnetization at the end of individual nanowires but in the case of long enough nanowires with strong shape anisotropy, a nearly single domain structure can be assumed ${ }^{10,14,18-20}$ and consequently, the orientation of magnetization in the whole nanowire can be so deduced from MFM contrast. In few cases, the magnetic contrast obtained in the MFM images has already been used to give a quantitative value of the magnetization ${ }^{14,21-23}$ in nanostructures. By analyzing the MFM imaging it is possible to get a comparison between bulk information provided by SQUID with surface information from individual nanowires. In addition, as discussed here in further detail, interaction between tip and magnetic surface may play an important role in the observed images.

In this work, we perform a systematic study to elucidate the most suitable method to evaluate the magnetization from the MFM images. The main achievement has been to derive a quantitative MFM imaging analysis by introducing a particular distribution function in the magnetic moments of individual nanowires (i.e., Gausssian and Lorenztian) from the observed MFM images, that allows one to obtain a direct quantitative distribution of macroscopic magnetization in the whole array.

\section{EXPERIMENTAL DETAILS}

The nanowire arrays have been prepared in our laboratory by using nanoporous alumina membranes as templates for subsequent filling the nanopores by electrodeposition technique. ${ }^{9,24,25}$ 


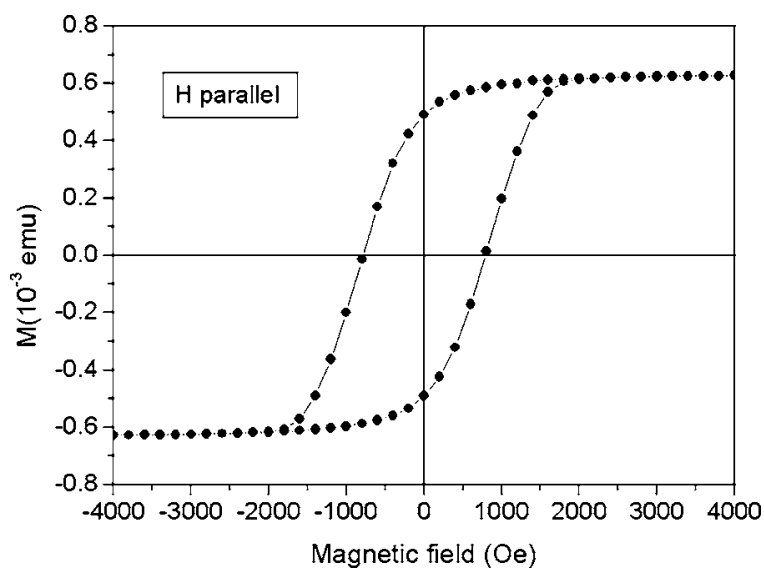

FIG. 1. Hysteresis loop measured by SQUID parallel to the wire direction.

In this work, the studied array consists of Ni polycrystalline wires [crystal size about $10 \mathrm{~nm}$ (Ref. 11)] with diameters about $35 \mathrm{~nm}$ and length of $1000 \mathrm{~nm}$ long. The ordered nanowires present hexagonal symmetry with a lattice parameter of $105 \mathrm{~nm}$.

Hysteresis loops with field parallel and perpendicular to the wires have been measured by SQUID magnetometer. The local magnetization distribution has been studied using a MFM equipment from Nanotec Electronica ${ }^{\mathrm{TM}}$. Such a system, working in noncontact mode allows us to acquire simultaneously the topography of the surface and the magnetic force gradient map. The MFM setup has been conveniently modified so that controlled magnetic field can be applied along different orientations (in plane of the membrane and parallel to the wires axis).

Particularly, MFM images of the array have been obtained at different remanence states after applying in situ magnetic field parallel to the wire direction. Standard MFM tips (with Co coating of $50 \mathrm{~nm}$ thick) have been premagnetized along the axial direction before each experiment. The MFM images have been analyzed by different methods in order to determine the remanent magnetization. In addition, the influence of the tip stray field on the remanence of the sample is evaluated.

\section{RESULTS AND DISCUSSION}

\section{A. Hysteresis loops}

Figure 1 shows the hysteresis loops performed on $\mathrm{Ni}$ nanowire array along axial (parallel to nanowires) direction. The magnetic information obtained from the hysteresis loops corresponds to a material with well defined axial easy axis with a coercive field of $H_{c}=780 \mathrm{Oe}$, larger than in $\mathrm{Ni}$ thin films. The remanent magnetization $M_{r}$ is about $0.8 M_{s}$.

The anisotropy of these nanowires arises mainly from shape anisotropy as a consequence of the large aspect ratio $R \sim 28$ and the small magnetostatic energy (the crystalline anisotropy is two orders of magnitude lower than the shape anisotropy $\left.{ }^{17}\right)$. In this situation, the individual nanowires can be considered as nearly single-domain structures with two stable states: the magnetic moment pointing to up or down directions. However, the behavior of the array as a whole

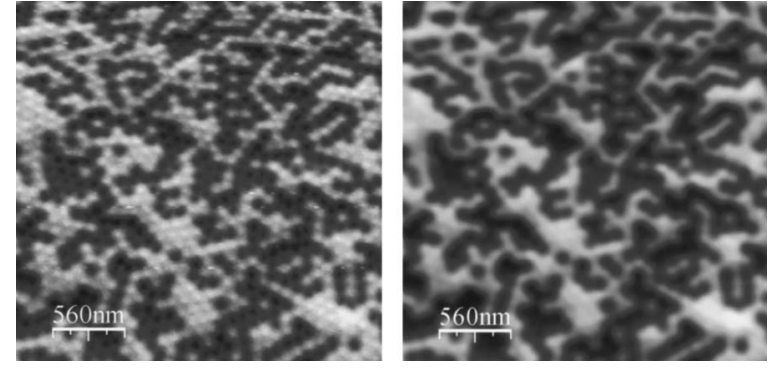

FIG. 2. (a) MFM image of a Ni nanowires array obtained at remanence state after demagnetizing under axial ac field. (b) Simulated MFM image corresponding to the MFM data in (a) distorted assuming a tip with a magnetic domain size of $140 \mathrm{~nm}$. Image size: $2.8 \times 2.8 \mu \mathrm{m}^{2}$.

differs from a bistable magnetic state system due to the magnetostatic interactions between nanowires. ${ }^{13,14,26}$

\section{B. Alternative methods for evaluating the remanent magnetization}

Considering individual nanowires to be single domain, positive and negative magnetic poles appear on the upper end of nanowires at the membrane surface. MFM images confirm this approach since the magnetic contrast presents only two states, in the following white and black contrasts, that correspond to nanowires with the magnetic moment oriented along up and down directions, respectively.

Figure 2(a) shows the MFM image of the array, at remanence state, after demagnetizing under a decreasing amplitude ac axial field. In this demagnetized state we observe nearly the same number of wires with magnetic moment aligned up and down where the nanowires with parallel magnetic moment arrange in chains or clusters. Due to the magnetostatic interaction among nanowires, the distribution of wires with opposite moments depends on the previous magnetic history.

By counting the number of wires pointing in each direction, the normalized remanent magnetization value, $M_{r} / M_{s}$, can be obtained as

$$
\frac{M_{r}}{M_{s}}=\frac{N_{b}-N_{w}}{N_{b}+N_{w}},
$$

where $M_{s}$ is the saturation magnetization and $N_{b}$ and $N_{w}$ are the number or black and white wires, respectively. The remanent magnetization calculated for the MFM image in Fig. 2 is $M_{r} / M_{s}=0.05$. The deviation from the zero value expected after demagnetization is due to the tip influence. For the standard tips and the tip-sample distances used in our experiments, the tip stray field is between ${ }^{27} 300$ and 500 Oe. The remanent value obtained from the MFM data is in agreement with these values and we will assume a permanent magnetic field of 400 Oe applied to the sample.

When the number of wires increases or the MFM images present poor definition due to the size of the domain in the tip apex (notice that the wire diameter is about $30 \mathrm{~nm}$ and the tip radius is $10 \mathrm{~nm}$ before the magnetic thin film deposition) it can be difficult to determine the number of wires pointing in each direction. For that reason, in this work, an alternative method based on the study of the MFM signal 

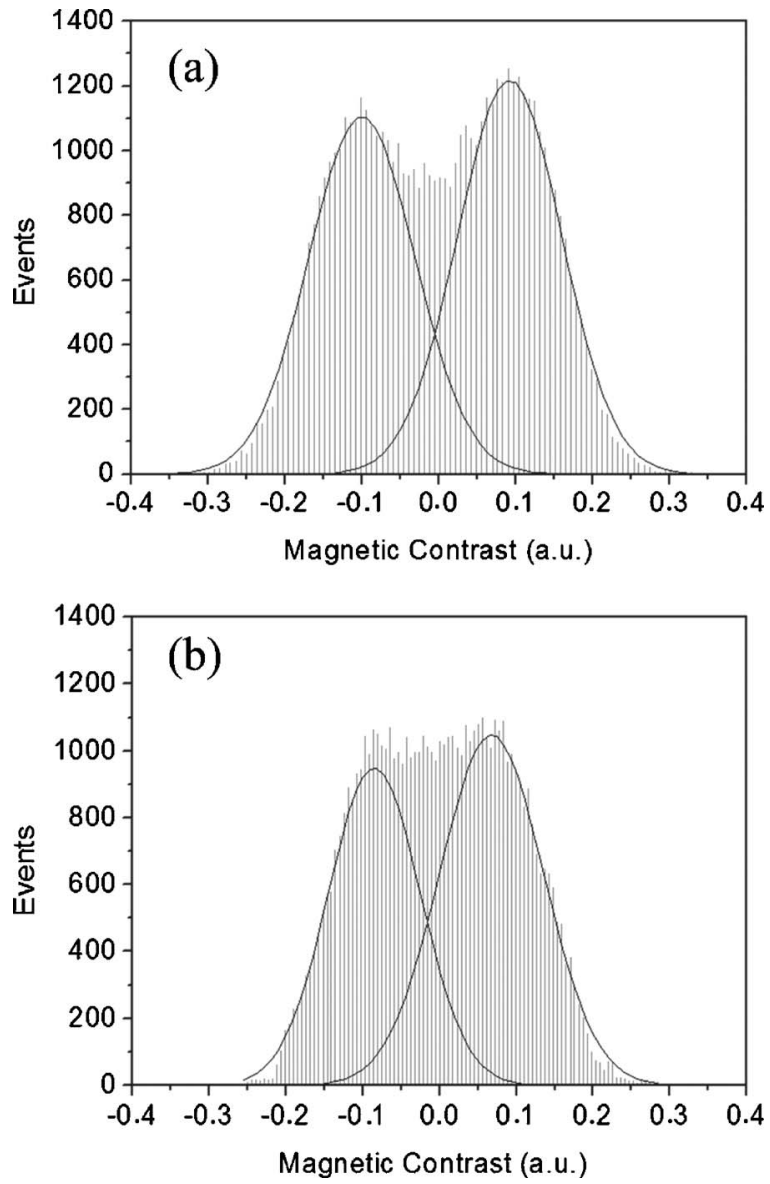

FIG. 3. Histograms corresponding to MFM data in Figs. 2(a) and 2(b), respectively, fitted to two Gaussian distribution functions.

distribution is proposed. In particular, the fitting of the MFM data to Gaussian or Lorentzian distribution functions will be considered.

First we have checked how the size of the domain at the end of the tip (responsible of the MFM contrast and resolution) affects the MFM data. We have simulated images assuming that the domain at the end of the tip has a size $(s)$ between 20 and $360 \mathrm{~nm}$. The MFM signal has been calculated taking into account only the perpendicular component of the forces and assuming an exponential decay of the interaction. The contribution of a differential element of the tip to the image in a particular position is

$$
\delta M \propto \exp \left(-\frac{x^{2}}{2 s}\right),
$$

where $s$ is the size of the tip and $x$ is the distance from the differential element to the center of the tip.

Figure 2(b) shows the simulated MFM image obtained assuming we have a tip with a domain size $s=140 \mathrm{~nm}$.

The histograms in Fig. 3 have been obtained from the MFM images in Figs. 2(a) and 2(b), respectively. The contrast distribution of the original image [Fig. 2(a)] presents two peaks corresponding to the nanowires with up (white color in the image and positive contrast values in the distribution) and down (black color in the image and negative contrast values in the distribution) magnetic moments. By increasing the domain size at the end of the tip, these peaks

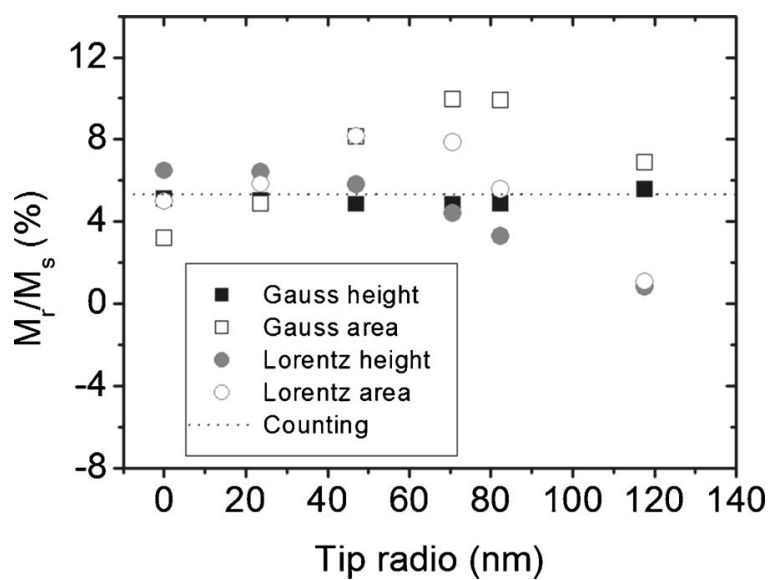

FIG. 4. $M_{r} / M_{s}$ evaluated by using the height or the area of the two Gaussian or Lorentzian distribution functions.

are distorted since the intermediate contrast increases. The contrast distribution has been fitted to Gaussian and Lorentzian distribution functions and we have used the peak height ( $G_{h}$ of the Gaussian distribution and $L_{h}$ of the Lorentzian distribution) as well as the peak area $\left(G_{a}\right.$ of the Gaussian distribution and $L_{a}$ of the Lorentzian distribution) to calculate the magnetization. Then, the values of $N_{x}$ in Eq. (1) will be $N_{x}=G_{x}$ or $N_{x}=L_{x}$ for the Gaussian or Lorentzian fittings, respectively.

For the original MFM image [Fig. 2(a)], the resulting magnetization values have been $M_{G h}=0.05, M_{G a}=0.04$, $M_{L h}=0.05$, and $M_{L a}=0.07$ where $M_{G h}$ is calculated by using the height of the Gaussian distribution, $M_{G a}$ the area of the Gaussian distribution, $M_{L h}$ the height of the Lorentzian distribution, and $M_{L a}$ the area of the Lorentzian distribution. The values calculated by using the heights of the Gaussian and Lorentzian fittings are in agreement with the remanent magnetization calculated previously by using the counting method $\left(M_{r} / M_{s}=0.05\right)$.

The same process has been repeated for the simulated images corresponding to the different final domain radius.

The results (see Fig. 4) indicate that the height of the Gaussian fitting is the best parameter to evaluate the magnetization when we have a large domain. In contrast, the evaluation of the magnetization by using the area integrated in the Gaussian or Lorentzian fittings must be disregarded. The reason is that MFM images could contain false contrast corresponding to the topography that increases the area of the black or white peaks. In addition, when the tip size increases, the edges of the MFM data distribution tend to decrease more sharply. In this sense, the Gaussian distribution fits our data better since it goes to zero more rapidly.

In the following, the counting method as well as the method based on the Gaussian fitting have been used to obtain the remanent magnetization values. Fitting to Gaussian distribution functions a maximum coefficient of determination $r^{2}=0.997$ is imposed. This method based on the Gaussian fitting can be useful to analyze uniaxial perpendicular magnetic materials by MFM and even more to calibrate the stray field of the MFM tips. ${ }^{28}$. 

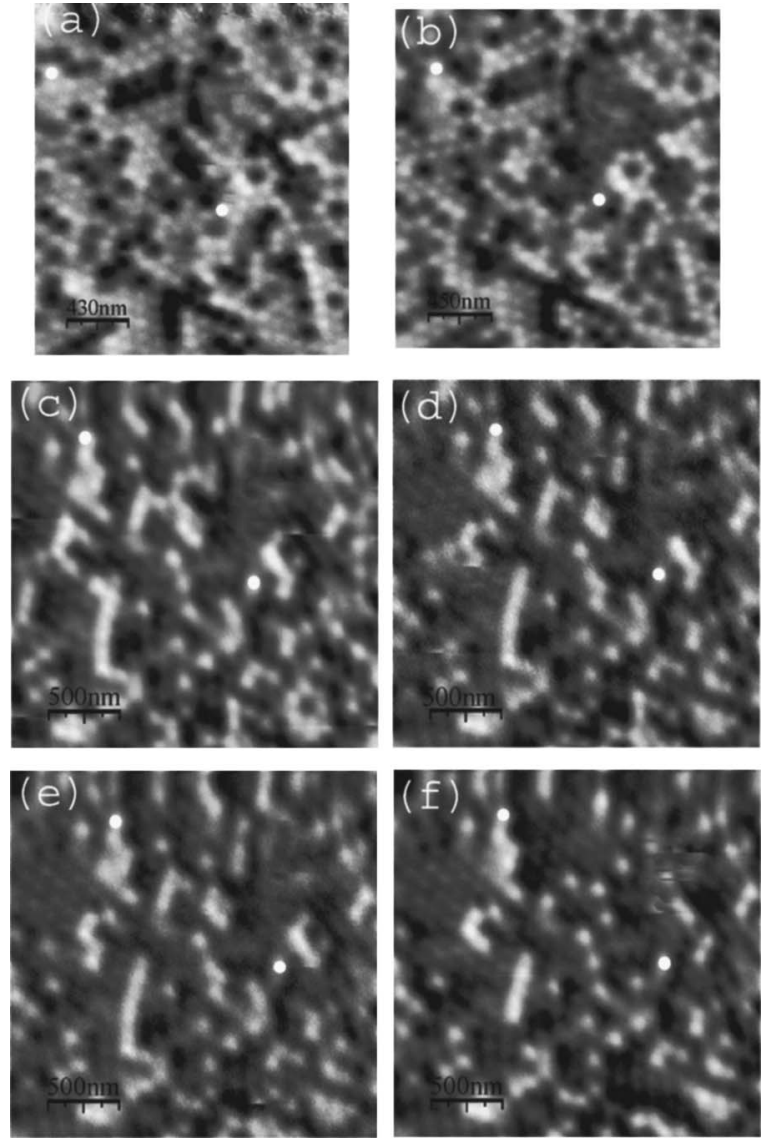

FIG. 5. MFM images obtained in the same region (a) initial state and after applying a parallel to the tip field of (b) 480 Oe, (c) 570 Oe, (d) 900 Oe, (e) $1230 \mathrm{Oe}$, and (f) $1560 \mathrm{Oe}$. Two white spots on top of two nanowires are used as a reference to follow the magnetic state evolution.

\section{Magnetization process measured by MFM}

By using the different methods to evaluate the magnetization with the MFM data, we calculate the changes in the magnetization regarding the previous magnetic history. This analysis allows us additionally to improve our knowledge about the magnetization process and the interaction between nanowires.

The images in Fig. 5 have been obtained by imaging the topography and the magnetic contrast of a particular region after applying different axial magnetic fields. Figure 5(a) corresponds to the sample in the initial remanent state. The images obtained after applying in situ parallel magnetic fields of 480, 570, 900, 1230, and 1560 Oe (see images from 5(b)-5(f), respectively) show us the magnetic state changes of the individual nanowires. Two white spots have been depicted on top of two nanowires to easily follow the nanowires magnetic state changes. The MFM tip was previously saturated with positive field, in the same direction as the subsequent applied magnetic field. An increment of black nanowires is observed since the magnetic field is applied in the same direction as the tip was previously saturated and the attractive forces produce a decrease of the resonance frequency.

Notice that groups of white adjacent nanowires reverse simultaneously [clearly observed by comparing the images $5(\mathrm{a})$ and 5(b) or the images 5(b) and 5(c)] against the dipolar
TABLE I. Remanent magnetization calculated by using two methods: counting the number of wires in each direction and using the height of the Gaussion fitting.

\begin{tabular}{ccc}
\hline \hline $\begin{array}{c}\text { Magnetic } \\
\text { field }\end{array}$ & $\begin{array}{c}M_{r} / M_{s} \\
(\text { Counting })\end{array}$ & $\begin{array}{c}M_{r} / M_{s} \\
\text { (Gaussion })\end{array}$ \\
\hline Initial state & $-0.20 \pm 0.02$ & $-0.15 \pm 0.057$ \\
+480 Oe & $+0.15 \pm 0.02$ & $+0.06 \pm 0.05$ \\
+570 Oe & $+0.37 \pm 0.02$ & $+0.30 \pm 0.05$ \\
+650 Oe & $+0.38 \pm 0.02$ & $+0.27 \pm 0.05$ \\
+740 Oe & $+0.40 \pm 0.02$ & $+0.27 \pm 0.05$ \\
+900 Oe & $+0.44 \pm 0.02$ & $+0.47 \pm 0.05$ \\
+1230 Oe & $+0.48 \pm 0.02$ & $+0.50 \pm 0.05$ \\
+1560 Oe & $+0.57 \pm 0.02$ & $+0.61 \pm 0.05$ \\
\hline
\end{tabular}

interaction between nanowires. ${ }^{16}$ We have also observed a wide range of switching fields that reveals the importance of the interaction between nanowires.

Deeper analysis of MFM images in Fig. 5 allows us to obtain quantitative information of the magnetic state of the array. Table I collects the remanent magnetization values deduced by counting the number of wires pointing at each direction as well as by fitting the MFM data to two Gaussian distribution functions corresponding to the positive and negative poles as shown above. The MFM image corresponding to the initial state presents more white than black
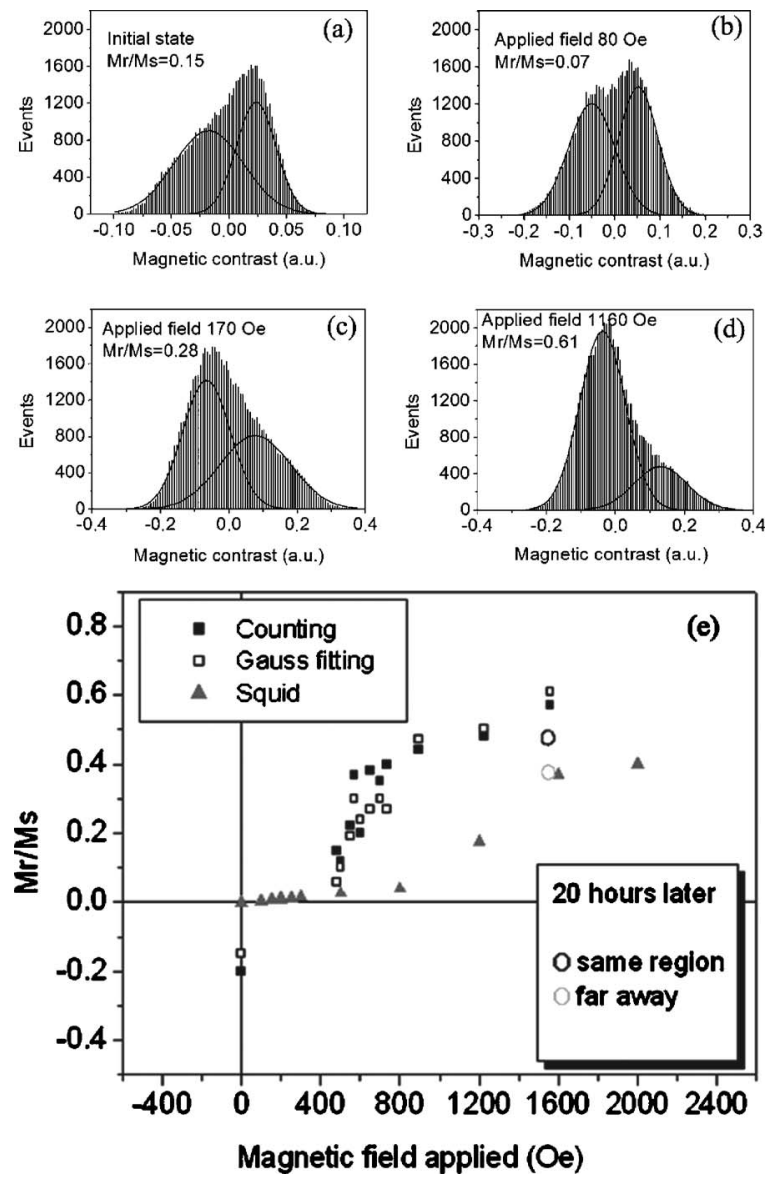

FIG. 6. [(a)-(d)] Magnetic contrast distributions corresponding to the MFM images (a)-(c) and (f) in Fig. 5. (e) Remanent magnetization vs minor loops amplitude. 


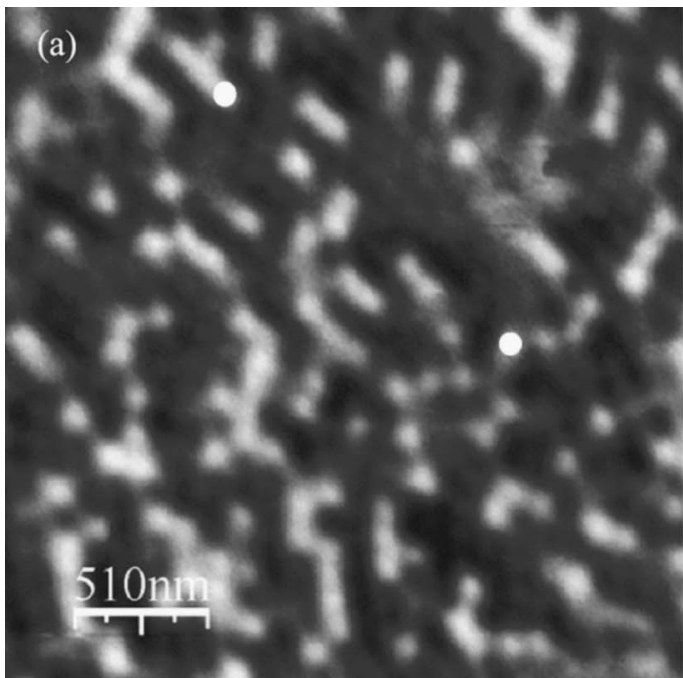

(b)

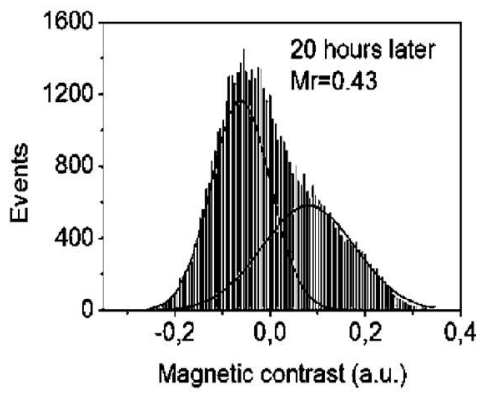

FIG. 7. (a) MFM image of the region shown in Fig. 5 obtained $20 \mathrm{~h}$ later. The white spots correspond to the same nanowires marked there. (c) Magnetic contrast distribution.

nanowires and the $M_{r}$ calculated by counting is about $-0.02 M_{s}$. However, in the remanence after applying a positive axial field, an increment of the black nanowires is observed and the $M_{r}$ values rise to $0.57 M_{s}$ when a magnetic field of 1560 Oe is applied. The analysis of the histograms in Fig. 6 gives us the same results. In the initial state, the distribution is shifted to the positive values (white nanowires). When the field is applied, the distribution moves to negative values. The results obtained from both methods are in good agreement; however, they do not fit to bulk minor hysteresis loops measured by SQUID as shown in Fig. 6(e).

In particular, the remanent magnetization is higher than expected seemingly due to the tip field contribution. Nevertheless, an extra tip field of 400 Oe (calculated value obtained previously from the MFM measurements in a demagnetized array) has already been taken into account in the applied field values. Assuming that the increment of the remanent magnetization is due to the further tip magnetic field and comparing the remanent values with the SQUID data, the tip field should be larger than $1000 \mathrm{Oe}$, too much bigger than the expected value for this kind of standard MFM tips.

In order to evaluate the effect of the proximity of the magnetic tip when the external magnetic field is applied, we have studied the same region and regions far away $20 \mathrm{~h}$ later (Fig. 7). We have found that the magnetic state relaxes and that the magnetic moment of some nanowires reverses decreasing the remanence magnetization from about $0.60 M_{s}$ to $0.40 M_{s}$ in the region studied previously. However, in regions tens and hundreds microns away, the remanence magnetization is much lower, about $0.35 M_{s}$. This result is in absolute agreement with the SQUID data revealing the usefulness of the MFM studies for obtaining the remanent magnetization values. However, the existence of an extra magnetic field originated by the presence of the tip must be taken into account. Since the tip field is always present, the main difference between regions is the position of the tip when the external magnetic field is applied. Systematically, the MFM probe is saturated in a VSM before every experiment applying an axial magnetic field. Then, the magnetic material of the tip presents a well defined remanence state where most of the domains are parallels to such magnetic field. Note here that the remanent magnetization of the probe is lower than the saturation magnetization and then domains in the opposite direction also appear. In the presence of an in situ external field, the magnetic material of the tip magnetizes increasing both the magnetic flux into the tip and its stray field. Due to the proximity of the tip, the local field just under the probe increases. Accordingly, we have observed that the effect of the opposite applied magnetic field is lower than expected.

\section{CONCLUSIONS}

In summary, MFM informs us about the magnetic state of individual nanowires and allows us to study locally the magnetization processes. By using two different methods for evaluating the amount of nanowires with the magnetization pointing in each direction, the remanent magnetization has been determined. Apart from the method of counting of black and white nanowires in the MFM images, the remanent magnetization can be calculated by using the magnetic contrast distribution functions. In particular, the fitting of the data to two Gaussian distributions reveals as a useful method that can be extended to the evaluation of the remanent magnetization of magnetic materials with perpendicular uniaxial anisotropy. The agreement between the results obtained from both methods and the SQUID data reveals the usefulness of the MFM technique for the quantitative study of the magnetic state of the materials. However, the tip influence must be taken into account in two senses: as a permanent field applied to the sample and as an extra magnetic field applied to the region just under the tip in the presence of an external magnetic field.

\section{ACKNOWLEDGMENTS}

The authors want to thank M. García for the SQUID measurements. This work was supported by projects CAM GR/MAT/0437/2004 and PTR95/0935/OP.

${ }^{1}$ Y. Martin, C. C. Williams, and H. K. Wickramasinghe, J. Appl. Phys. 61, 4723 (1987).

${ }^{2}$ J. J. Sáenz, N. García, P. Grütter, E. Meyer, H. Heinzelmann, R. Wiesendanger, L. Rosenthaler, H. R. Hidber, and H. J. Güntherodt, J. Appl. Phys. 62, 4293 (1987) 
${ }^{3}$ A. Hubert and R. Schäfer, Magnetic Domains (Springer-Verlag, Berlin, 1998).

${ }^{4}$ A. Asenjo, D. García, J. M. García, C. Prados, and M. Vázquez, Phys. Rev. B 62, 6538 (2000).

${ }^{5}$ H. Masuda and K. Fukuda, Science 268, 1466 (1995).

${ }^{6}$ L. Piraux, J. M. George, J. F. Despres, C. Leroy, E. Ferain, R. Legras, K. Ounadjela, and A. Fert, Appl. Phys. Lett. 65, 2484 (1994).

${ }^{7}$ See Proceedings of the International Workshop on Magnetic Wires, San Sebastián, Spain, special issue of J. Magn. Magn. Mater. 1-2, 249 (2002).

${ }^{8}$ R. O'Barr, S. Y. Yamamoto, S. Schultz, W. Xu, and A. Scherer, J. Appl. Phys. 81, 4730 (1997)

${ }^{9}$ K. Nielsch, R. B. Wehrspohn, J. Barthel, J. Kirschner, U. Gösele, S. F. Fischer, and H. Kronmüller, Appl. Phys. Lett. 79, 1360 (2001).

${ }^{10}$ R. Ferre, K. Ounadjela, J. M. George, L. Piraux, and S. Dubois, Phys. Rev. B 56, 14066 (1997).

${ }^{11}$ W. Wernsdorfer, B. Doudin, D. Mailly, K. Hassebach, A. Benoit, J. Meier, J.-Ph. Ansermet, and B. Barbara, Phys. Rev. Lett. 77, 1873 (1996).

${ }^{12}$ P. M. Paulus, F. Luis, M. Kröll, G. Schmid, and L. J. de Jongh, J. Magn. Magn. Mater. 224, 180 (2001).

${ }^{13}$ C. A. Ross et al., Phys. Rev. B 65, 144417 (2002).

${ }^{14}$ T. G. Sorop, C. Untiedt, F. Luis, M. Kröll, M. Rasa, and L. J. de Jongh, Phys. Rev. B 67, 014402 (2003).

${ }^{15}$ M. Vázquez et al., Physica B 343, 395 (2004).
${ }^{16}$ M. Vázquez, C. Ramos, E. Vassallo, M. Jaafar, and A. Asenjo, Trans. Magn. Soc. Jpn. 5, 157 (2005).

${ }^{17}$ M. E. Schabes, J. Magn. Magn. Mater. 95, 249 (1991).

${ }^{18}$ K. Ounadjela, R. Ferre, L. Louail, J. M. George, J. L. Maurice, L. Piraux, and S. Dubois, J. Appl. Phys. 81, 5455 (1997).

${ }^{19}$ J.-E. Wegrowe, D. Kelly, A. Franck, S. E. Gilbert, and J.-Ph. Ansermet, Phys. Rev. Lett. 82, 3681 (1999).

${ }^{20}$ R. Hertel, J. Appl. Phys. 90, 5752 (2001).

${ }^{21}$ C. Haginoya, S. Heike, M. Ishibashi, K. Nakamura, and K. Koike, J. Appl. Phys. 85, 8327 (1999).

${ }^{22}$ K. Nielsch et al., J. Magn. Magn. Mater. 249, 234 (2002).

${ }^{23}$ C. A. Ross, M. Farhoud, M. Hwang, J. I. Smith, M. Redjdal, and F. B. Humphrey, J. Appl. Phys. 89, 1310 (2001).

${ }^{24}$ J. M. García, A. Asenjo, M. Vázquez, P. Aranda, and E. Ruiz-Hiztky, IEEE Trans. Magn. 36, 2981 (2000).

${ }^{25}$ M. Vázquez, K. Pirota, M. Hernández-Vélez, V. M. Prida, D. Navas, R. Sanz, F. Batallán, and J. Velázquez, J. Appl. Phys. 95, 6642 (2004).

${ }^{26}$ M. Vázquez, M. Hernandez-Velez, K. Pirota, A. Asenjo, D. Navas, J. Velázquez, P. Vargas, and C. Ramos, Eur. Phys. J. B 40, 489 (2004).

${ }^{27}$ See Technical Specifications provided by the probe companies.

${ }^{28}$ M. Jaafar, A. Asenjo, and M. Vázquez, IEEE Trans. Nanotechnol. (submitted). 